\title{
Hippocampal Formation Supports Conditioning to Memory of a Context
}

\author{
Jerry W. Rudy, Ruth M. Barrientos, and Randall C. O’Reilly \\ University of Colorado, Boulder
}

\begin{abstract}
It has been proposed that contextual fear conditioning depends on 2 processes: (a) construction of a conjunctive representation of the features that make up the context and (b) association of the representation with shock. Support for this view comes from studies indicating that prior exposure to the conditioning context facilitates contextual fear conditioning supported by immediate shock. Thus, conditioning produced by immediate shock is to the memory representation of the preexposed context, which is activated by retrieval cues associated with this context. The authors' experiments support this interpretation and indicate that this process depends on an intact hippocampal formation. These results support the hypothesis that the hippocampal formation supports contextual fear conditioning by storing a conjunctive representation of context.
\end{abstract}

The importance of fear conditioning to the neurobiology of memory emerged when it was reported that fear to the context or place in which shock occurred (contextual fear) and fear to a discrete auditory cue paired with the shock were differentially dependent upon the hippocampal formation (Kim \& Fanselow, 1992; Phillips \& LeDoux, 1992; Selden, Everitt, Jarrard, \& Robbins, 1991). Damage to the hippocampal formation impaired contextual but not auditory-cue fear conditioning. The selectivity of the result suggested that the study of contextual fear conditioning would provide a valuable method for studying the mechanisms by which the hippocampus contributes to memory and is now commonly used for this purpose.

Several theorists have argued that contextual fear conditioning depends on two independent learning processes: The rat (a) constructs and stores a conjunctive representation of the independent features of the context and (b) associates that representation with shock (see Fanselow \& Rudy, 1998; Rudy, 1996; Rudy \& Morledge, 1994; Young, Bohenek, \& Fanselow, 1994). In this framework, it is the acquisition of the conjunctive representation that requires the hippocampus. The associative process does not.

There is considerable support for the view that rats can acquire a representation of context, independent of associating it with shock. This support comes from a range of experiments that have demonstrated what we call the context preexposure facilitation effect - the fact that preexposure to the conditioning context the day before a context-shock experience can enhance the low levels of fear contextual conditioning that otherwise would occur (Fanselow, 1990; Pugh et al., 2000; Pugh, Tremblay, Fleshner, \& Rudy, 1997; Rudy, Kuwagama, \& Pugh, 1999; Rudy \& Pugh, 1998; Westbrook, Good, \& Kiernan, 1994).

Fanselow (1990) was the first researcher to report this effect. He did so by studying the so-called immediate-shock effect. Although

Jerry W. Rudy, Ruth M. Barrientos, and Randall C. O'Reilly, Department of Psychology, University of Colorado, Boulder.

This research was supported by National Institutes of Health Grant RO1 MH61316-01 to Jerry W. Rudy and Randall C. O'Reilly.

Correspondence concerning this article should be addressed to Jerry W. Rudy, Department of Psychology, 345 UCB, University of Colorado, Boulder, Colorado 80309. E-mail: jrudy@psych.colorado.edu the rat will display fear to the context if placed in the conditioning chamber for about $1 \mathrm{~min}$ before the shock, if it is shocked immediately after being placed in the chamber, it will subsequently show almost no freezing. Fanselow's important observation was that briefly exposing the rat to the context a day prior to the shock session significantly facilitated contextual fear conditioning normally supported by immediate shock (see also Kiernan \& Westbrook, 1993; Westbrook et al., 1994).

The theory about why preexposure to the context can facilitate the amount of conditioning normally associated with immediate shock and its dependency on the hippocampus needs elaboration. There are four assumptions that should be made explicit.

The preexposure period allows the rat to acquire a conjunctive representation of the context automatically as a product of exploring the environment. During the immediate-shock phase, the rat samples a subset of the features of the preexposed context, and this subset retrieves or activates the conjunctive memory representation. This is called pattern completion.

Conditioning that is produced by the immediate shock is to the activated memory representation of the preexposed context. The hippocampal formation is critical to the acquisition of a conjunctive representation and the pattern completion process it supports. It is important to briefly discuss the concept of a conjunctive representation and to distinguish it from a features representation. A context or a place is composed of many independent features (e.g., floor texture, odor, illumination, sound level, and so on). Thus, when it explores a particular context, an animal can store the unconnected representations of these independent features, or these co-occurring features can be bound together in a unique representation. A conjunctive representation contains the bound co-occurring features (see O'Reilly \& Rudy, 2001; Rudy \& O'Reilly, 2001).

Because a conjunctive memory representation contains the bound features of an experience, it is thought to be able to support the pattern completion process: When an input pattern of features $(\mathrm{A}, \mathrm{B}, \mathrm{C}, \mathrm{D}, \mathrm{E})$ is conjoined into a memory representation, the presentation of a subset of features (e.g., A, E) will activate the memory for the entire input pattern (Marr, 1971; McNaughton \& Morris, 1987; O’Reilly \& McClelland, 1994; O’Reilly \& Rudy, 2001; Rudy \& O’Reilly, 1999, 2001). 
The ability of a subset of features to reactivate the whole pattern is important for cued recall of stored memories, and it is the ability of the hippocampus to support conjunctive representations and pattern completion that is thought to link this brain region to declarative memory in humans (Squire, 1992; Teyler \& DiScenna, 1986). For example, in his review of this literature, Squire suggested that "in the present account the possibility of later retrieval is provided by the hippocampal system because it has bound together the relevant cortical sites. A partial cue that is later processed through the hippocampus is able to reactivate all of the sites and thereby accomplish retrieval of the whole memory" ( $p$. 224).

The importance of pattern completion to the context preexposure facilitation effect was in fact demonstrated by Fanselow (1990) in his original analysis of the immediate-shock effect. He reported that preexposure did not eliminate the immediate-shock effect if the rats indeed were shocked immediately after being placed in the context. Facilitation occurred only when the rats were in the context for about $8 \mathrm{~s}$. Fanselow argued that for preexposure to the context to facilitate conditioning supported by immediate shock, the rat must spend enough time in the context just before the shock to allow a subset of cues to retrieve the memory representation of the context prior to the shock.

Note that a strong implication of this analysis of the context preexposure facilitation effect is that conditioning supported by immediate shock is to the activated memory representation of the preexposed context. We have, in fact, recently provided strong support for this conclusion and for the key underlying assumption that rats acquire conjunctive representation of the context that can support the pattern completion on which memory activation depends (Rudy \& O'Reilly, 2001). Specifically, Rudy and O'Reilly showed that immediately shocked rats actually conditioned to the memory of the preexposure environment and not to the environment where shock conditioning actually took place (see the introduction to Experiment 2 for a more detailed description).

In summary, the existing literature supports three basic claims about the processes that support the contextual preexposure facilitation effect: (a) rats learn about the context independent of its association with shock, (b) they acquire a conjunctive representation of context, and (c) they condition to a memory representation of context. However, what needs to be demonstrated is that this learning depends on a contribution from the hippocampal formation. Thus, the purpose of the experiments that we present here was to determine if the conjunctive learning that takes place when the rat is exposed to a novel context requires a contribution from the hippocampus.

\section{Experiment 1}

The purpose of the first experiment was to determine whether the basic context preexposure facilitation effect depends on the hippocampal formation. We noted that Fanselow (1990) reported that context preexposure facilitated conditioning supported by immediate shock. Rats had to be in the context for a few seconds before the shock for this effect to occur. Recently, however, we have developed a methodology that produces the context preexposure facilitation effect, even though rats were shocked immediately upon placement in the context (Rudy \& O'Reilly, 2001). Our strategy was to provide training that would attach the representation of the context to the transport cues preceding placement of the rat in the context. In this way, we hoped that the transport cues would activate the representation of the context prior to the shock. We did this by giving the rat multiple trials in which they were transported, in a distinct light-sealed black bucket, to the context. If rats were transported to the preexposed context in the bucket and immediately shocked, they then displayed robust contextual fear conditioning. However, if they were transported to the preexposed context in a transport cage not associated with that context, the context preexposure facilitation effect was not observed. We used this methodology to examine the role of the hippocampus in the acquisition of conjunctive representations of contexts.

In our experiments, we attempted to restrict our lesions to the dorsal hippocampal formation region, instead of damaging the entire hippocampal formation, because it has been suggested that any impaired contextual fear conditioning produced by damage to ventral hippocampus could be due to disrupting inputs to the nucleus accumbens, thereby altering the rat's exploratory behavior that is essential for it to normally sample its environment (Maren, 1999; Richmond et al., 1999) or by damaging the amygdala (Anagnostaras, Gale, \& Fanselow, 2001). We made axon-sparing neurotoxic lesions because it has been suggested (Maren, 1999) that impaired contextual fear conditioning associated with electrolytic damage to the dorsal hippocampus may result from damage to fibers of passage that traverse the dorsal hippocampus from ventral subiculum to brain structures critical to exploration (nucleus accumbens; Burns, Annett, Kelley, Everitt, \& Robbins, 1996) and fear (amygdala; Canteras \& Swanson, 1992).

Rats with damage to the hippocampus or control rats were preexposed either to the conditioning context or to another context. All rats were then transported to the conditioning context and shocked immediately. They were tested for fear of that context the next day. We expected that control rats, preexposed to the conditioning context, would display more contextual fear than rats exposed to a different context, thereby demonstrating a context preexposure facilitation effect. If our theory is correct, however, damage to the hippocampus should reduce this facilitation.

\section{Method}

Subjects. Subjects were adult male Long-Evans derived rats bred the University of Colorado. They were housed in groups of 3-4 subjects in plastic cages and maintained on a 12-hr light-dark cycle. Preexposure, conditioning, and testing occurred between 7 a.m. and 10:30 a.m.

Surgery and histology. Under Nembutal anesthesia $(50 \mathrm{mg} / \mathrm{kg})$, rats were given bilateral infusions of $N$-methyl-D-aspartate $(20 \mu \mathrm{g} / \mu \mathrm{l}, 0.1$ $\mu 1 /$ min, $0.4 \mu 1$, Sigma Chemical, St. Louis, MO) at four sites within the dorsal hippocampus. Coordinates for the first and second sites, respectively, were as follows: 2.8 and $4.2 \mathrm{~mm}$ posterior to bregma, 1.6 and 2.6 $\mathrm{mm}$ lateral to the midline, and 4.0 and $3.6 \mathrm{~mm}$ ventral to the skull surface. Rats were given at least 10 days to recover before the behavioral procedures were initiated. All procedures were in accordance with the University of Colorado Animal Care and Use Committee. At the end of the experiment, the rats were anesthetized with Nembutal and decapitated. Brains were removed and frozen. Coronal frozen sections (40 $\mu \mathrm{m}$ thick) were sliced through the extent of the hippocampus with a cryostat at $-19{ }^{\circ} \mathrm{C}$, mounting every third section. Sections were stained with cresyl violet and examined by light microscopy to visually verify dorsal hippocampal lesions.

Apparatus. Conditioning occurred in two identical Igloo ice chests (54 $\mathrm{cm}$ long $\times 30 \mathrm{~cm}$ wide $\times 27 \mathrm{~cm}$ high) with white interiors. During a conditioning session, a Plexiglas chamber sat on the rod floor that was used to deliver the unconditioned stimulus. The unconditioned stimulus, a 
2-s, 0.65-mA shock, was delivered through a removable floor of stainless steel rods $1.5 \mathrm{~mm}$ in diameter, spaced $1.2 \mathrm{~cm}$ center to center. Each rod was wired to a shock generator and scrambler (Model 8240415-SS, Lafayette Instruments, Lafayette, IN). The rods and floor of each chamber were cleaned before each rat was trained or tested.

Conditioned fear was assessed by measuring conditioned freezing-the rat's natural defensive response to anticipated danger (Blanchard \& Blanchard, 1969). During the contextual fear test, rats were placed in a conditioning chamber and observed for $6 \mathrm{~min}$. Using a time-sampling procedure, every $10 \mathrm{~s}$ the observers judged each rat as either freezing or active at the instant the sample was taken. Freezing was defined as the absence of visible movement except for respiration. In our laboratory, the correlation between observers exceeds .95 . At the time of testing, neither of the two observers had knowledge of the subjects' treatment condition.

Design and training procedures. The general design for this experiment is displayed in the top of Figure 1. Rats were exposed to either the conditioning context, Context A, or the control context, Context B. Context A was a transparent rectangular chamber $(26 \mathrm{~cm}$ long $\times 21 \mathrm{~cm}$ wide $\times 24$ $\mathrm{cm}$ high) placed on a steel rod floor that was used to deliver shock. It was placed inside an ice chest with a white interior that was illuminated by a 6 -watt clear bulb. The ice chest door was open, and the entire room was illuminated by two 60 -watt bulbs. Context $\mathrm{B}$ was an opaque mouse cage

A
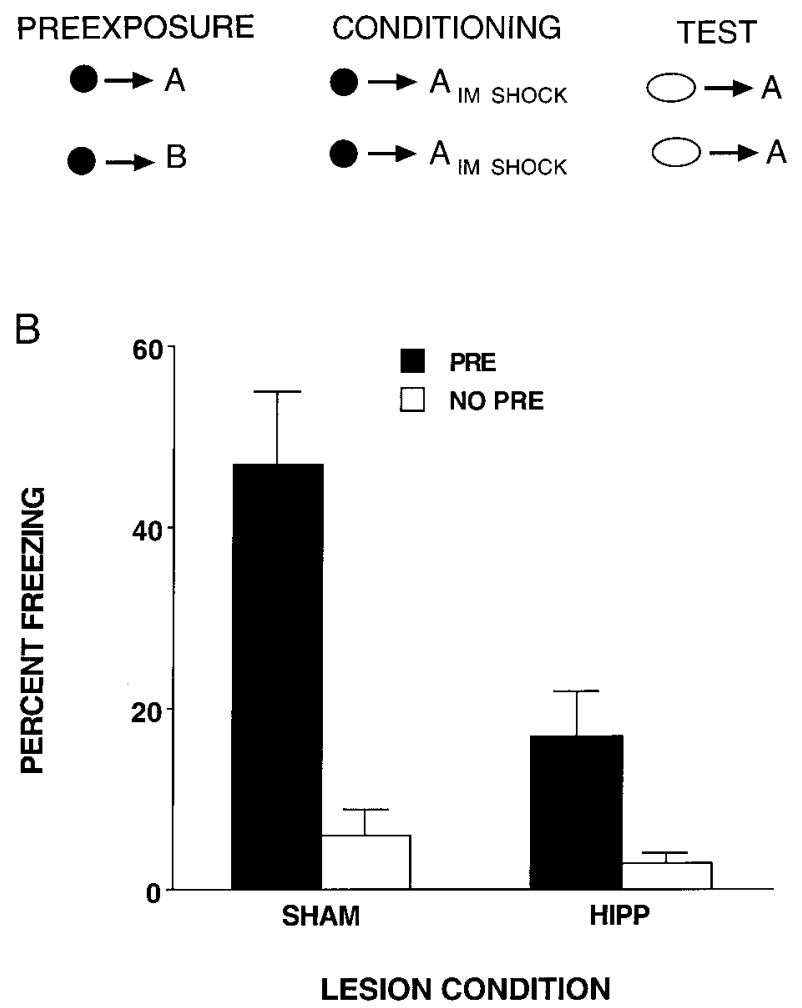

Figure 1. A: The three phases of Experiment 1. The black circle represents the black bucket used for transport during preexposure; the white oval represents the perforated pan used for transport during testing. During preexposure, different rats were preexposed to either Context A (transparent rectangular chamber) or Context B (mouse cage). On the conditioning day, all rats were transported to Context $\mathrm{C}$ (dimly lit chamber) in the black bucket and immediately shocked (IM SHOCK). B: Mean ( \pm SEM) percentage of freezing in the contextual fear test as a function of lesion condition. $\mathrm{PRE}=$ preexposed; $\mathrm{NO} \mathrm{PRE}=$ non-preexposed; HIPP $=$ hippocampal-lesioned rats.
( $26 \mathrm{~cm}$ long $\times 16 \mathrm{~cm}$ wide $\times 12 \mathrm{~cm}$ high) with a stainless steel top and was located in a different room. During preexposure, rats were transported to the chambers in a light-sealed black ice bucket. Transportation required about $20 \mathrm{~s}$. The lid was placed on the bucket when rats were taken to the chambers but was removed when they were returned to their home cage. Each rat received a single 4-min preexposure on Day 1. On Day 2, all rats were exposed to their respective context four times. Each exposure was approximately $40 \mathrm{~s}$. Exposures 1 and 2 and Exposures 3 and 4 were separated by $2 \mathrm{~min}$. A 10-min interval separated Exposures 2 and 3. On Day 3, all rats were transported to the conditioning context and immediately shocked. On Day 4, all rats were transported in a perforated metal pan to the conditioning chamber where their freezing response was observed for $6 \mathrm{~min}$.

\section{Results and Discussion}

Histology. Histological reconstructions from rats representing the largest and smallest extent of damage to the dorsal hippocampal formation are presented in Figure 2. In general, these lesions were complete and included substantial damage to the dorsal subiculum. In rats with less complete damage, there was sparing in cells of the CA2, and in some cases CA3, subfields of the hippocampus.

Contextual fear. As expected, immediate shock produced very little conditioning in rats that were not preexposed to the conditioning context (i.e., the immediate-shock effect). In contrast, preexposure to the conditioning context (Context A) significantly enhanced the freezing displayed by control rats, but did not enhance conditioning by rats with damage to dorsal hippocampus (see Figure 1). A two-factor analysis of variance (ANOVA) revealed an interaction between the preexposure condition and the lesion condition, $F(1,31)=5.13, p<.03$. Analyses of the simple effects indicated that control rats preexposed to Context $\mathrm{A}(n=$ 10) displayed more freezing than rats with damage to dorsal hippocampus that had been preexposed to Context A $(n=7), F(1$, $31)=12.00, p<.01$, and control rats preexposed to Context $\mathrm{A}$ displayed more freezing than control rats preexposed to Context $\mathrm{B}$ $(n=8), F(1,31)=24.60, p<.01$. Rats with damage to dorsal hippocampus preexposed to Context A did not differ from rats preexposed to Context $\mathrm{B}(n=9)$.

This experiment replicated our previous finding (Rudy \& O'Reilly, 1999) that even if the rat is immediately shocked, multiple preexposures facilitate strong contextual fear conditioning. This implies that the transport cues, via the pattern completion process, were able to activate the memory representation of the preexposed context so that it could associate with the immediate shock. That damage to the hippocampal formation significantly reduced the context preexposure facilitation effect is consistent with the idea that the hippocampus is essential to the processes that mediate this effect.

\section{Experiment 2}

A strong implication of the results of Experiment 1 (see also Rudy \& O'Reilly, 2001), which is also the underlying theory behind the experiment, is that the physical cues present at the time of shock are less important for the development of conditioned fear than the memory representation that is active. Indeed, if the pattern completion interpretation is correct, then the preexposed rats in the previous experiment were conditioned to the memory representation activated by the transport environment and not to the physical 


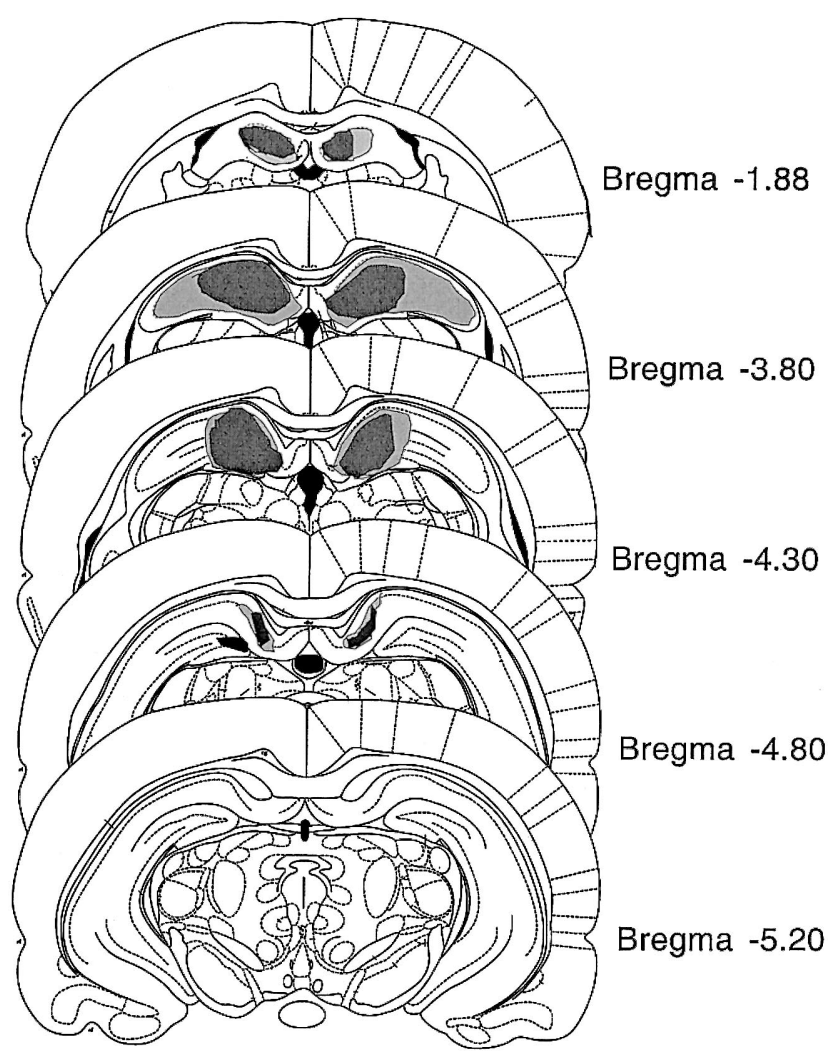

Figure 2. Representation of the largest and smallest lesions of the dorsal hippocampal formation. The gray area represents the extent of the largest lesion, and the black area represents the extent of the smallest lesion. From The Rat Brain in Stereotaxic Coordinates (4th ed., Figures 26, 35, 37, 39, and 40), by G. Paxinos and C. Watson, 1998, San Diego, CA: Academic Press. Copyright 1998 by Academic Press. Adapted with permission.

cues present at the time of shock. Rudy and O'Reilly, in fact, provided strong support for the idea that conditioning produced by immediate shock was to the recalled memory representation of the preexposed environment. To do this, we established a transport container-context association through preexposure, and then used this transport container to bring rats into a novel context for immediate shock conditioning (see Figure 3).

The rationale for this design was as follows. Because rats had no prior experience with the novel context, they would not associate the immediate shock with it. However, if the transport cues, via pattern completion, activated the memory of the preexposure context, this representation would associate with the immediate shock. Note that this analysis predicts that rats would display more conditioning to the preexposed context than to the context in which the immediate shock was experienced. Rudy and O'Reilly (2001) evaluated and confirmed this prediction. Rats displayed virtually no conditioning to the shock context but displayed strong fear to the preexposed context.

In Experiment 2, we used this design to further explore the contribution the hippocampus makes to processes underlying the context preexposure facilitation effect. Following the training depicted in Figure 3, rats were tested in either the preexposed context or the context in which the shock occurred. Given our prior results and the previous analysis, we expected that (a) intact rats would display more contextual fear to the preexposed context than to the context in which shock occurred, (b) damage to the hippocampus would have no influence when rats were tested in the shocked context (neither control nor lesioned rats should display fear in the shocked context), but (c) damage to the hippocampus would impair contextual fear expressed in the preexposed context.

\section{Method}

The procedure for Experiment 2 is illustrated in Figure 3. All rats received a total of nine exposures to either Context A or Context B: four on Day 1 and five on Day 2. The first exposure lasted 4 min. Thereafter, all exposures were approximately $40 \mathrm{~s}$. On Day 2, between preexposure Trials 3 and 5, each rat was also placed twice (for $2 \mathrm{~min}$ ) in a perforated metal pan. On Day 3, the rats were conditioned. They were taken from their home cage in the black bucket, placed in Context $\mathrm{C}$, and shocked immediately. On Day 4, rats from each group were transported in the perforated metal pan to either the shock context, Context $\mathrm{C}$, or to the preexposure context, Context A. Their freezing was scored for 6 min. Context A was a transparent rectangular chamber $(26 \mathrm{~cm}$ long $\times 21 \mathrm{~cm}$ wide $\times 24 \mathrm{~cm} \mathrm{high})$ that sat on a Plexiglas floor. Context $\mathrm{B}$ was the mouse cage. Context $\mathrm{C}$ was constructed from clear Plexiglas $(26 \mathrm{~cm}$ long $\times 21 \mathrm{~cm}$ wide $\times 10 \mathrm{~cm}$ high), but it sat on the steel rod floor in the ice chest. There was no lighting in the conditioning room when rats were placed in Context $\mathrm{C}$, and the interior of the ice chest was dimly illuminated with a red light bulb. Illumination was set so that there was just enough light to carry out conditioning and to permit scoring. Note that Context $\mathrm{C}$ was designed to be dissimilar to Context A.

\section{Results and Discussion}

The histology results were essentially the same as those obtained in Experiment 1. Figure 3B presents the results for the rats that were tested in Context $\mathrm{C}$, the context in which immediate shock was delivered. There were 6 subjects in each condition represented in Figure 3B. Note that regardless of the preexposure or lesion condition, these rats displayed almost no fear. Figure $3 \mathrm{C}$ shows the results for rats tested in Context $\mathrm{A}$, the preexposed context. It shows that control rats preexposed to Context $\mathrm{A}$ and shocked in Context $\mathrm{C}$ displayed robust contextual fear conditioning in Context $\mathrm{A}$ in comparison to rats preexposed to Context B. In contrast, rats with bilateral damage to the dorsal hippocampus showed little conditioned freezing, regardless of where they were preexposed. A two-factor ANOVA revealed an interaction between preexposure condition and lesion condition, $F(1$, $25)=4.70, p<.03$. Analysis of the simple effects indicated that control rats preexposed to Context A $(n=10)$ displayed more freezing than lesioned rats $(n=8)$ preexposed to Context A, $F(1$, $25)=9.00, p<.01$, and that control rats preexposed to Context A displayed more freezing than control rats $(n=5)$ that had been preexposed to Context B, $F(1,25)=10.00, p<.01$. Rats with damage to the hippocampus and preexposed to Context A did not differ from rats preexposed to Context $\mathrm{B}(n=6)$.

These results replicate our previous finding (Rudy \& O'Reilly, 2001) that under these training conditions intact, immediately shocked rats display more conditioned fear in the preexposed context than in the context in which the shock actually occurred. This result provides strong evidence that during immediate shock, rats condition to the memory representation activated by the transport cues and not to the physical features of the environment in which the shock occurred. The results of this experiment also indicate that the hippocampal formation is critical to this outcome 
A

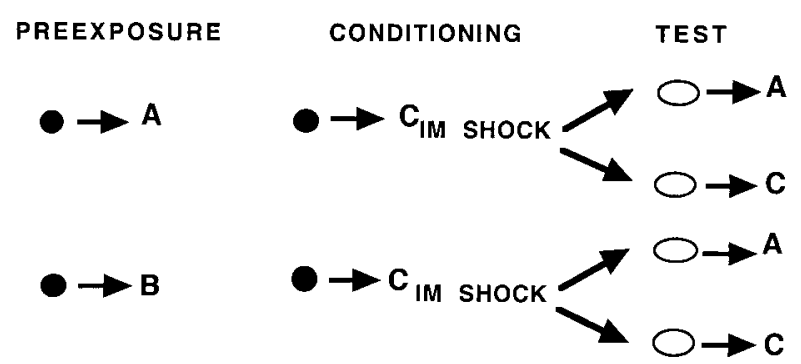

B

C
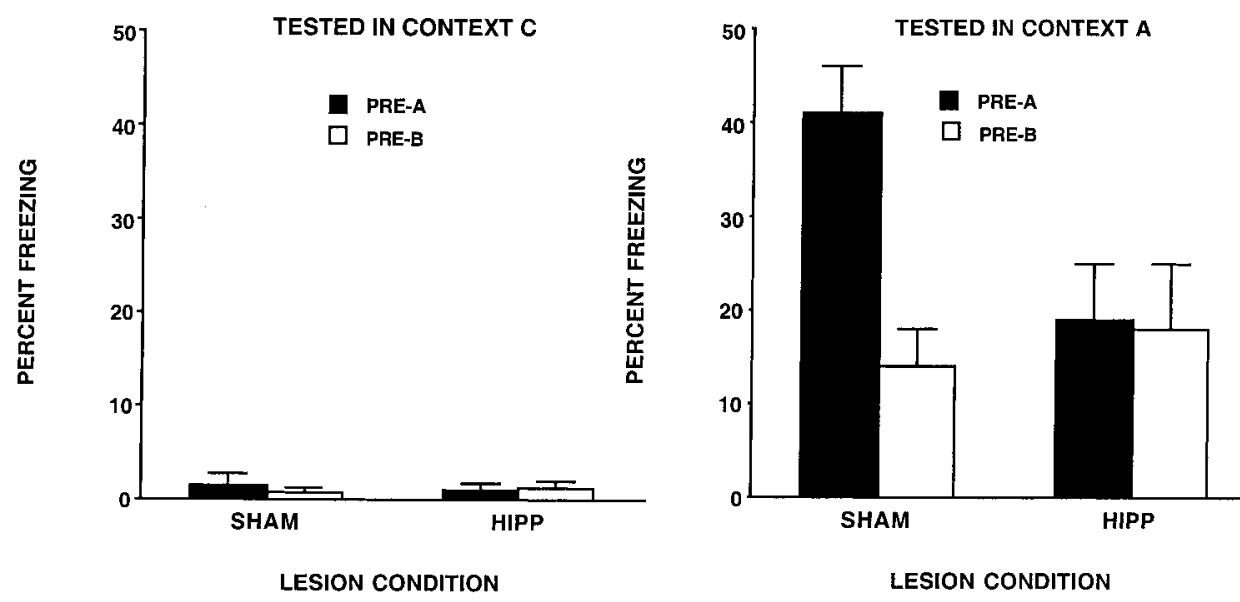

Figure 3. A: The three phases of Experiment 2. The black circle represents the black bucket used for transport during preexposure; the white oval represents the perforated pan used for transport during testing. During preexposure, different rats were preexposed to either Context A (transparent rectangular chamber) or Context B (mouse cage). On the conditioning day, all rats were transported to Context $\mathrm{C}$ (dimly lit chamber) in the black bucket and immediately shocked (IM SHOCK). Half the rats from the two preexposure conditions were tested in Context $\mathrm{A}$, and half were tested in Context C. B and C: Mean ( \pm SEM) percentage of freezing in the contextual fear test as a function of preexposure condition. $\mathrm{PRE}=$ preexposed; HIPP $=$ hippocampal-lesioned rats.

because rats with damage to this brain region were impaired on this task.

\section{Experiment 3}

One could argue that the results of Experiments 1 and 2 were not due to the impact of hippocampal formation damage on rats learning about context per se, but instead to either (a) their inability to associate context features with shock or (b) their processes that express fear were impaired. However, neither of these alternatives seems likely when considered against the recent literature indicating that neurotoxic damage to the dorsal hippocampus prior to conditioning does not impair contextual fear conditioning if the dorsal hippocampus is damaged prior to conditioning (Frankland, Cestari, Filipkowski, McDonald, \& Silva, 1998; Maren, Aharonov, \& Fanselow, 1997; Richmond et al., 1999). Nevertheless, to rule these alternatives out, we evaluated the effect of the lesion on a more standard form of contextual fear conditioning.

\section{Method}

One week after the completion of Experiment 2, all rats from Experiment 2 were transported to a different conditioning context in the perforated metal pan where they received three shocks. The intershock interval was $2 \mathrm{~min}$, and the first shock occurred $2 \mathrm{~min}$ after the rats were placed in the conditioning context. Freezing was scored during the 2 min that preceded the first shock and during the 2 min after the first and second shocks. The next day the rats were transported to the conditioning chamber and tested for $8 \mathrm{~min}$. The conditioning chamber was clear Plexiglas $(26 \mathrm{~cm}$ long $\times 21 \mathrm{~cm}$ wide $\times 10 \mathrm{~cm}$ high) and sat on the steel rod floor in the illuminated ice chest.

\section{Results and Discussion}

As shown in Figure 4, damage to the hippocampal formation did not affect the acquisition of contextual fear conditioning. Regardless of lesion condition, on the conditioning day, rats displayed little freezing during the 2-min period that preceded the first shock. 

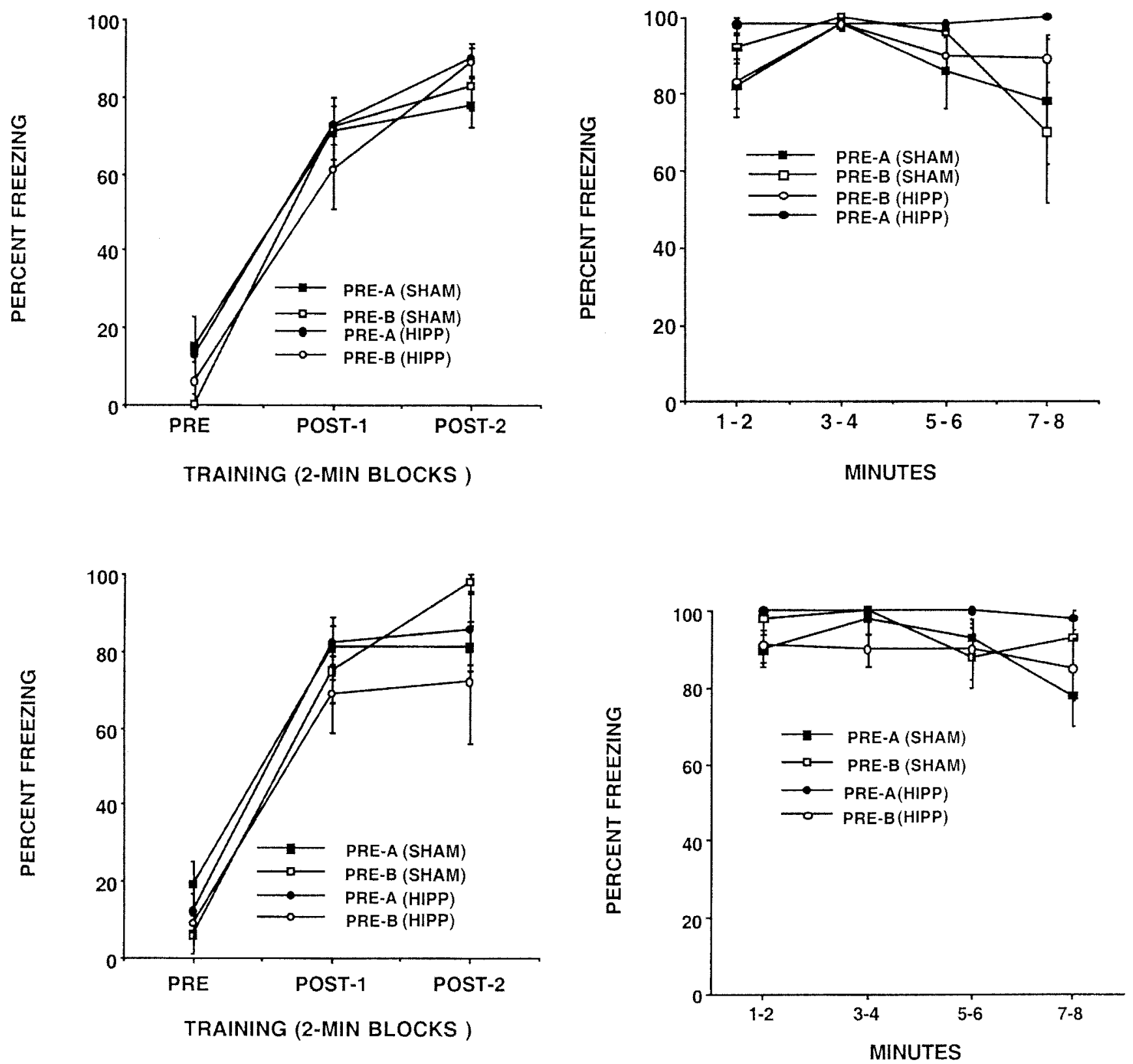

Figure 4. Mean $( \pm S E M)$ percentage of freezing to the context in Experiment 3. The top panels present the data for rats tested in Context $\mathrm{C}$ (the conditioning context, a dimly lit chamber) in Experiment 2. The bottom panels present the data for rats tested in Context A (the preexposed context, a transparent rectangular chamber) in Experiment 2. The left panels present the results obtained during the conditioning session, and the right panels present the results of the fear test the next day. PRE $=$ the first $2 \mathrm{~min}$ of testing that occurred prior to the first shock; POST-1 = the 2-min period after the first shock; POST-2 = the 2-min period after the second shock; HIPP $=$ hippocampal-lesioned rats.

Following the shock, the level of freezing increased dramatically, but there were no differences among the groups either on the conditioning day or during the fear test the next day.

These results indicate that rats with dorsal hippocampal lesions are capable of freezing, making it unlikely that the results of either Experiment 1 or Experiment 2 were due to their inability to express fear. They also indicate that rats with dorsal hippocampal lesion can associate shock with the features of the context and, therefore, strengthen our conclusion that the results of the previous experiments occurred because the hippocampal formation makes an important contribution to the rat's acquisition of a conjunctive representation of the features of the conditioning context. However, it should be acknowledged that because the rats in Experiment 3 received three shocks on the training day, whereas rats trained in the immediate-shock experiment received only a single shock, that these data are not as strong as they could be. Never- 
theless, the data are in agreement with results reported by Maren et al. (1997) and Richmond et al. (1999) that damage to the dorsal hippocampal formation prior to training has no effect on the acquisition of contextual fear conditioning.

Two recent studies (Barrientos et al., in press; Barrientos, O'Reilly, \& Rudy, in press) provide additional support for the view that the hippocampus contributes to the storage of the context representation. In these studies, either the protein synthesis inhibitor Anisomycin or the proinflammatory cytokine interleukin $1 \beta$ (IL-1 $\beta$ ) was injected into the dorsal hippocampus following preexposure to the context to block the consolidation of the context memory. Compared with rats injected with the vehicle, rats injected with Anisomycin or IL- $1 \beta$ displayed a significantly reduced context preexposure facilitation effect when given an immediate shock. Because the hippocampus was intact during the immediateshock phase and the test phase, this result cannot be due to impaired associative learning (the context-shock association) or the inability of the rat to express fear at the time of testing.

\section{General Discussion}

Our results (Experiments 1 and 2) are consistent with the reports that preexposure dramatically increased contextual fear normally associated with immediate shock (Fanselow, 1990; Rudy \& O'Reilly, 2001; Westbrook et al., 1994). In addition, these results add the important result that this context preexposure facilitation effect depends on an intact hippocampal formation. Moreover, consistent with the recent literature, we found that damage to dorsal hippocampus did not impair performance produced by a standard contextual fear conditioning paradigm (Experiment 3). Together with the existing literature, these findings support the following theoretical conclusions:

1. When a normal rat explores a novel context, it automatically acquires a conjunctive representation of its features.

2. This representation can be activated by a subset of the features that belong to the conjunction (pattern completion).

3. Conditioning that is produced by the immediate shock is to the activated memory representation of the preexposed context.

4. The hippocampal formation provides a critical neural substrate for the mechanism that supports these storage and retrieval processes.

5. Fear to a context can be supported by extrahippocampal brain regions.

The first conclusion derives primarily from our finding that the context facilitation effect requires that the configuration of features that make up the preexposed context be identical to the configuration of features that make up the conditioning context. Preexposure to the features in other configurations does not facilitate contextual fear conditioning (Rudy \& O'Reilly, 1999). The second conclusion is implied by the finding that immediate shock can support strong contextual fear conditioning if (a) the rat has been preexposed to the context and (b) immediate shock is preceded by transport cues associated with the preexposed context (see also Rudy \& O'Reilly, 2001). The third conclusion is forced by the finding that immediately shocked rats display more fear to the preexposure context associated with the transport cues than they do to the novel context in which shock actually was experienced (Experiment 2; Rudy \& O'Reilly, 2001). This result could only occur if the shock was associated with the memory representation of the context retrieved by the transport cues. It could not be the product of stimulus generalization of fear established to the conditioning context because the rats displayed no fear to the conditioning context. The fourth conclusion is supported by the fact that damage to the hippocampal formation significantly reduced the context preexposure facilitation effect (Experiments 1 and 2). The fifth conclusion is supported by our findings that, although damage to the dorsal hippocampal formation eliminated the context preexposure facilitation effect, it did not impair contextual fear conditioning under more standard training (Experiment 3; see also Maren et al., 1997; Richmond et al., 1999).

Our results indicate that the hippocampus contributes to contextual fear conditioning by supporting the acquisition of a conjunctive representation of the context. As noted, however, damage to the hippocampal formation does not always impair contextual fear conditioning. For example, there are several reports (including Experiment 3) that neurotoxic lesions of dorsal hippocampus prior to training can have no effect on contextual fear conditioning (Maren et al., 1997; Richmond et al., 1999). Damage to the entire hippocampus prior to conditioning or damage to ventral hippocampus (Maren et al., 1997; Richmond et al., 1999) has been reported to impair contextual fear, but it also significantly influences the rat's exploratory behavior. This finding leaves open the possibility that impaired fear conditioning associated with damage to ventral hippocampus might just reflect the effect of the lesion on encoding instead of memory processing supported by the hippocampus. It also has been argued that the effect of hippocampal damage on contextual fear is a performance effect that is due to the disruption of the processes that express fear via the freezing response (Gewirtz, McNish, \& Davis, 2000).

In isolation, such results question the idea that the hippocampus contributes to contextual fear conditioning. However, it should also be appreciated that when neurotoxic damage to dorsal hippocampus occurs after conditioning, contextual fear conditioning is severely impaired (see Anagnostaras et al., 2001; Maren et al., 1997). This strongly implies that the hippocampus contributes to contextual fear conditioning in the normal rat.

It is not surprising that the contribution the hippocampus makes to contextual fear is not straightforward. This is because several theoretical frameworks linking the hippocampus to memory assume that the collection of features that provide a space, place, or context for experience can be represented in two ways: (a) a features view, which assumes that the context is represented as a set of independent features or elements that each can be entered into association with events, and (b) a conjunctive or mapping view, which assumes that the individual features are combined into a unitary representation that is different from the sum of its parts (Anagnostaras et al., 2001; Fanselow, 2000; Maren et al., 1997; Nadel \& Willner, 1980; Nadel, Willner, \& Kurz, 1985; O’Reilly \& Rudy, 2001; Rudy \& O'Reilly, 1999, 2001; Rudy \& Sutherland, 1994, 1995). Within these frameworks, the hippocampus is thought to support the acquisition of the conjunctive representation and extrahippocampal areas are assumed to support the representation of the features and their linkages.

In such frameworks, there is no a priori reason to suppose that damage to the hippocampus necessarily should impair contextual fear conditioning because the independent features of the context themselves could associate with the shock and support conditioned fear. The finding that anterograde damage to the hippocampus does not always impair contextual fear conditioning is consistent with this view. Thus, the finding that retrograde damage to the 
hippocampus severely compromises contextual fear conditioning is important because it provides strong evidence that the hippocampal formation is involved in contextual fear conditioning. In part, this is because it renders the performance deficit interpretation unlikely. Regardless of whether the hippocampus is damaged before or after training, by a performance account, the rat's expression of fear should be compromised (see also Anagnostaras, Maren, \& Fanselow, 1999).

On the basis of the results of studies of the anterograde and retrograde effects of damage to the hippocampal formation on contextual fear conditioning, Maren et al. (1997) have argued that the feature and conjunctive representations of context exist in a hierarchical relationship: The intact rat is biased to use the conjunctive representation over the features representation. Thus, when conditioned prior to the lesion, the rat associates shock with the hippocampal-dependent conjunctive representation at the expense of conditioning to context features represented in brain regions outside the hippocampal formation. So, when the hippocampus is damaged after conditioning, contextual fear is severely impaired because the intact rat did not condition to the features and the hippocampal support for the conjunctive representation is no longer available. However, when the hippocampus is damaged prior to conditioning, the features are free to associate with shock and impaired fear conditioning may not be observed.

Thus, the analysis of the contribution the hippocampus makes to contextual fear conditioning is complicated by the fact that the brain can make use of two different representations of context: a features representation that does not depend on the hippocampus and a conjunctive representation that does (Fanselow, 1990; Maren et al., 1997; O'Reilly \& Rudy, 2001; Rudy \& O'Reilly, 1999, 2001). Because contextual fear conditioning can be supported by associations between the feature representations and shock, there will be a parameter space in which contextual fear conditioning will not be impaired by damage to the hippocampus.

It is because extrahippocampal regions can support featurebased contextual fear conditioning that our work has focused on the context preexposure facilitation effect. Conjunctive representation theories of hippocampal function assume that it contributes to contextual fear conditioning primarily by constructing a unitary representation of the stimulus environment. From this perspective, it is important to examine the contribution the hippocampus makes to contextual fear conditioning by using a methodology that separates the learning of a context representation from associating that representation with the shock. It is also important to use a methodology that enables one to determine that this learning produced a conjunctive representation of the context. The context preexposure methodology clearly separates learning about context from the shock association phase, and it has proved possible to demonstrate that as a consequence of preexposure, rats indeed acquire a conjunctive representation of the environment (Rudy \& O'Reilly, 1999). Consequently, in demonstrating that damage to the hippocampus eliminates the context preexposure facilitation effect, we have provided strong support for the idea that the hippocampal formation contributes to contextual fear conditioning by storing a conjunctive representation of context.

By focusing on the context preexposure facilitation effect, we also have provided strong support for an important implication of conjunctive representation theory and the pattern completion process it is assumed to support. Specifically, as applied to the immediate-shock experiment, these ideas imply that preexposure enhances contextual fear conditioning produced by immediate shock because conditioning is to the memory representation of the preexposed context that is activated at the time of the shock and not to the immediate sensory features of the conditioning context. The results of Experiment 2 provide strong evidence for this idea.

It has been argued that the reexperience of time and place during retrieval differentiates one form of human declarative memory, episodic memory, from other forms of conscious memory (e.g., Eldridge, Knowlton, Furmanski, Bookheimer, \& Engel, 2000). Nonverbal organisms may engage in conscious recollection, but researchers cannot yet measure this process directly. So, any link between animal memory and human declarative memory requires a mechanistic theory that connects a particular task to memory. A number of theorists (Marr, 1971; O'Reilly \& Rudy, 2001; Rudy \& O’Reilly, 1999, 2001; Squire, 1992; Teyler \& DiScenna, 1986) have argued that conjunctive representations and pattern completion supported by the hippocampus are the mechanistic basis for human declarative memory. We think that the theoretical mechanisms of pattern completion supported by conjunctive representations provide an important way to connect animal and human declarative memory because they do not require the subject to make a verbal response to indicate that a memory has been recollected.

We have established that the context preexposure facilitation effect depends on the rat acquiring a conjunctive representation that can be activated by the pattern completion process and that the facilitation effect depends on an intact hippocampus. Consequently, the context preexposure methodology provides an ideal animal model for studying the biological mechanisms that support declarative memory.

\section{References}

Anagnostaras, S. G., Gale, G. D., \& Fanselow, M. S. (2001). Hippocampus and contextual fear conditioning: Recent controversies and advances. Hippocampus, 11, 8-17.

Anagnostaras, S. G., Maren, S., \& Fanselow, M. S. (1999). Temporally graded retrograde amnesia of contextual fear after hippocampal damage in rats: Within-subjects examination. Journal of Neuroscience, 19, $1106-1114$.

Barrientos, R. M., Higgins, E., Sprunger, D., Wakins, L. R., Rudy, J. W., \& Maier, S. F. (in press). Memory for context is impaired by a post context exposure injection of interleukin-1 beta into dorsal hippocampus. Behavioural Brain Research.

Barrientos, R. M., O’Reilly, R. C., \& Rudy, J. W. (in press). Memory for context is impaired by injecting anisomycin into dorsal hippocampus following context exploration. Behavioural Brain Research.

Blanchard, R. J., \& Blanchard, D. C. (1969). Crouching as an index of fear. Journal of Comparative Physiology and Psychology, 67, 370-375.

Burns, L. H., Annett, L., Kelley, A. E., Everitt, B. J., \& Robbins, T. W. (1996). Effects of lesions to amygdala, ventral subiculum, medial prefrontal cortex, and nucleus accumbens on the reaction to novelty: Implication for limbic-striatal interactions. Behavioral Neuroscience, 110, $60-73$.

Canteras, N. S., \& Swanson, L. W. (1992). Projections of the ventral subiculum to the amygdala, septum, and hypothalamus: A PHAL anterograde tract-tracing study in the rat. Journal of Computational Neurology, 324, 180-194.

Eldridge, L. L., Knowlton, B. J., Furmanski, C. S., Bookheimer, S. Y., \& Engel, S. A. (2000). Remembering episodes: A selective role for the hippocampus during retrieval. Nature Neuroscience, 3, 1149-1152.

Fanselow, M. S. (1990). Factors governing one trial contextual conditioning. Animal Learning \& Behavior, 18, 264-270. 
Fanselow, M. S. (2000). Contextual fear, gestalt memories, and the hippocampus. Behavioural Brain Research, 110, 73-81.

Fanselow, M. S., \& Rudy, J. W. (1998). Convergence of experimental and developmental approaches to animal learning and memory processes. In T. Carew, R. Menzel, \& C. Shatz (Eds.), Mechanistic relationships between development and learning: Beyond metaphor (pp. 15-28). New York: Wiley.

Frankland, P. W., Cestari, V., Filipkowski, R. K., McDonald, R. J., \& Silva, A. J. (1998). The dorsal hippocampus is essential for context discrimination but not for contextual conditioning. Behavioral Neuroscience, 112, 863-874.

Gewirtz, J. C., McNish, K. A., \& Davis, M. (2000). Is the hippocampus necessary for contextual fear conditioning? Behavioural Brain Research, 110, 83-95.

Kiernan, M. J., \& Westbrook, R. F. (1993). Effects of exposure to a to-be-shocked environment upon the rat's freezing response: Evidence for facilitation, latent inhibition, and perceptual learning. Quarterly Journal of Experimental Psychology: Comparative and Physiological Psychology, 46(B), B271-B288.

Kim, J. J., \& Fanselow, M. S. (1992, May 1). Modality-specific retrograde amnesia of fear. Science, 256, 675-677.

Maren, S. (1999). Neurotoxic or electrolytic lesions of the ventral subiculum produce deficits in the acquisition and expression of Pavlovian fear conditioning in rats. Behavioral Neuroscience, 113, 283-290.

Maren, S., Aharonov, G., \& Fanselow, M. S. (1997). Neurotoxic lesions of the dorsal hippocampus and Pavlovian fear conditioning in rats. Behavioural Brain Research, 88, 261-274.

Marr, D. (1971). Simple memory: A theory for archicortex. Philosophical Transactions of the Royal Society of London, Series B, Biological Sciences, 262, 23-81.

McNaughton, B. L., \& Morris, R. G. M. (1987). Hippocampal synaptic enhancement and information storage within a distributed memory system. Trends in Neuroscience, 10, 408-415.

Nadel, L., \& Willner, J. (1980). Context and conditioning: A place for space. Physiology \& Behavior, 8, 218-228.

Nadel, L., Willner, J., \& Kurz, E. M. (1985). Cognitive maps and environmental context. In P. Balsam \& A. Tomie (Eds.), Context and learning (pp. 385-406). Hillsdale, NJ: Erlbaum.

O'Reilly, R. C., \& McClelland, J. L. (1994). Hippocampal conjunctive encoding, storage, and recall: Avoiding a trade-off. Hippocampus, 4, 661-682.

O'Reilly, R. C., \& Rudy, J. W. (2001). Conjunctive representations in learning and memory: Principles of cortical and hippocampal function. Psychological Review, 108, 311-345.

Paxinos, G., \& Watson, C. (1998). The rat brain in stereotaxic coordinates (4th ed.). San Diego, CA: Academic Press.

Phillips, R. G., \& LeDoux, J. E. (1992). Differential contribution of amygdala and hippocampus to cued and contextual fear conditioning. Behavioral Neuroscience, 106, 274-285.

Pugh, C. R., Johnson, J. D., Martin, D., Rudy, J. W., Maier, S. F., \& Watkins, L. R. (2000). Human immunodeficiency virus-1 coat protein gp120 impairs contextual fear conditioning: A potential role in AIDS related learning and memory impairments. Brain Research, 861, 8-15.

Pugh, C. R., Tremblay, D., Fleshner, M., \& Rudy, J. W. (1997). A selective role for corticosterone in contextual-fear conditioning. Behavioral Neuroscience, 111, 503-511.

Richmond, M. A., Yee, B. K., Pouzet, B., Veenman, L., Rawlins, J. N., Feldon, J., \& Bannerman, D. M. (1999). Dissociating context and space within the hippocampus: Effects of complete, dorsal, and ventral excitotoxic hippocampal lesions on conditioned freezing and spatial learning. Behavioral Neuroscience, 113, 1189-2203.

Rudy, J. W. (1996). Postconditioning isolation disrupts contextual conditioning: An experimental analysis. Behavioral Neuroscience, 110, 238 246.

Rudy, J. W., Kuwagama, K., \& Pugh, C. R. (1999). Isolation reduces contextual but not auditory-cue fear conditioning: A role for endogenous opioids. Behavioral Neuroscience, 113, 316-323.

Rudy, J. W., \& Morledge, P. (1994). Ontogeny of contextual fear conditioning in rats: Implications for consolidation, infantile amnesia, and hippocampal system function. Behavioral Neuroscience, 108, 227-234.

Rudy, J. W., \& O'Reilly, R. C. (1999). Contextual fear conditioning, conjunctive representations, pattern completion, and the hippocampus. Behavioral Neuroscience, 113, 867-880.

Rudy, J. W., \& O'Reilly, R. C. (2001). Conjunctive representations, the hippocampus, and contextual fear conditioning. Cognitive, Affective, \& Behavioral Neuroscience, 1, 66-82.

Rudy, J. W., \& Pugh, C. R. (1998). Time of conditioning selectively influences contextual fear conditioning: Further support for a multiplememory systems view of fear conditioning. Journal of Experimental Psychology: Animal Behavioral Processes, 24, 316-324.

Rudy, J. W., \& Sutherland, R. J. (1994). The memory coherence problem, configural associations and the hippocampal system. In D. Schacter \& E. Tulving (Eds.), Memory systems (pp. 119-147). Cambridge, MA: MIT Press.

Rudy, J. W., \& Sutherland, R. J. (1995). Configural association theory and the hippocampal formation: An appraisal and reconfiguration. Hippocampus, 5, 375-389.

Selden, N. R., Everitt, B. J., Jarrard, L. E., \& Robbins, T. W. (1991). Complementary roles for the amygdala and hippocampus in aversive conditioning to explicit and contextual cues. Neuroscience, 42, 335-350.

Squire, L. R. (1992). Memory and the hippocampus: A synthesis from findings with rats, monkeys, and humans. Psychological Review, 99, 195-231.

Teyler, T. J., \& DiScenna, P. (1986). The hippocampal memory indexing theory. Behavioral Neuroscience, 100, 147-154.

Westbrook, R. F., Good, A. J., \& Kiernan, M. J. (1994). Effects of the interval between exposure to a novel environment and the occurrence of shock on the freezing responses of rats. Quarterly Journal of Experimental Psychology: Comparative and Physiological Psychology, 47(B), B427-B446.

Young, S. L., Bohenek, D. L., \& Fanselow, M. S. (1994). NMDA processes mediate anterograde amnesia of contextual fear conditioning induced by hippocampal damage: Immunization against amnesia by context preexposure. Behavioral Neuroscience, 108, 19-29.

Received November 6, 2001

Revision received February 5, 2002

Accepted February 11, 2002 